OPEN ACCESS

Edited by:

Sixue Chen,

University of Florida, USA

Reviewed by:

David Twell,

University of Leicester, UK

Mengmeng Zhu,

The Pennsylvania State University,

USA

${ }^{*}$ Correspondence:

Tai Wang,

Key Laboratory of Plant Molecular

Physiology, Institute of Botany,

Chinese Academy of Sciences,

20 Nanxincun, Xiangshan, Haidianqu,

Beijing 100093, China

twang@ibcas.ac.cn

Specialty section:

This article was submitted to

Plant Systems and Synthetic Biology,

a section of the journal

Frontiers in Plant Science

Received: 14 April 2015

Accepted: 16 May 2015

Published: 02 June 2015

Citation:

Lu Y, Wei L and Wang T (2015)

Methods to isolate a large amount

of generative cells, sperm cells

and vegetative nuclei from tomato

pollen for "omics" analysis.

Front. Plant Sci. 6:391.

doi: 10.3389/fp/s.2015.00391

\section{Methods to isolate a large amount of generative cells, sperm cells and vegetative nuclei from tomato pollen for "omics" analysis}

\author{
Yunlong Lu ${ }^{1,2}$, Liqin Wei ${ }^{1}$ and Tai Wang ${ }^{1 *}$ \\ ${ }^{1}$ Key Laboratory of Plant Molecular Physiology, Institute of Botany, Chinese Academy of Sciences, Beijing, China, \\ ${ }^{2}$ University of Chinese Academy of Sciences, Beijing, China
}

The development of sperm cells (SCs) from microspores involves a set of finely regulated molecular and cellular events and the coordination of these events. The mechanisms underlying these events and their interconnections remain a major challenge. Systems analysis of genome-wide molecular networks and functional modules with highthroughput "omics" approaches is crucial for understanding the mechanisms; however, this study is hindered because of the difficulty in isolating a large amount of cells of different types, especially generative cells (GCs), from the pollen. Here, we optimized the conditions of tomato pollen germination and pollen tube growth to allow for long-term growth of pollen tubes in vitro with SCs generated in the tube. Using this culture system, we developed methods for isolating GCs, SCs and vegetative cell nuclei (VN) from just-germinated tomato pollen grains and growing pollen tubes and their purification by Percoll density gradient centrifugation. The purity and viability of isolated GCs and SCs were confirmed by microscopy examination and fluorescein diacetate staining, respectively, and the integrity of VN was confirmed by propidium iodide staining. We could obtain about 1.5 million GCs and 2.0 million SCs each from $180 \mathrm{mg}$ initiated pollen grains, and 10 million VN from $270 \mathrm{mg}$ initiated pollen grains germinated in vitro in each experiment. These methods provide the necessary preconditions for systematic biology studies of SC development and differentiation in higher plants.

Keywords: Solanum lycopersicum, generative cell, sperm cell, vegetative nuclei, isolation, Percoll density gradient centrifugation

\section{Introduction}

During the development of sperm cells (SCs, male gamete) from microspores in higher plants, the microspore generated from diploid microsporocytes via meiosis first undergoes asymmetric mitosis to produce a larger vegetative cell (VC) and a smaller generative cell (GC) embedded in the VC. Thereafter, the VC exits the cell cycle and has potential to generate a polarly growing pollen tube; the GC enters further mitosis to produce two SCs for double fertilization (McCormick, 1993; Twell et al., 1998; Twell, 2011). Depending on the plant species, GC mitosis occurs before anthesis or in growing pollen tubes; therefore, released mature pollen at anthesis is tricellular in some species such as Oryza sativa, Zea mays, and Arabidopsis thaliana (Berger and Twell, 2011) or bicellular in other species such as Lilium brownii and Solanum lycopersicum. This development 
process involves a set of fine-tuned molecular and cellular events and the coordination of these events, such as cell cycle regulation, cell differentiation and fate determination, genome stability, and epigenetic reprogramming. Although genetic studies have functionally identified many important genes involved in plant SC development, such as DUO1, DUO3, DAZ1, and DAZ2 (Borg et al., 2009, 2011, 2014; Brownfield et al., 2009a,b; Twell, 2011), the mechanisms underlying these events and their interconnections remain a major challenge for plant science. Systematic "omics" studies of the development process are essential for understanding the mechanisms.

"Omics" studies of pollen from several plants including Arabidopsis and rice have provided insights into the molecular mechanisms of pollen development (Rutley and Twell, 2015). During postmeiotic development from microspores, pollen express a set of specific transcripts; the total number of transcripts expressed is decreased, but the proportion of pollen-specific or preferential transcripts is increased (Honys and Twell, 2004; Wang et al., 2008; Wei et al., 2010). The composition and expression profile of miRNAs expressed in developing pollen differs from those in sporophytes, and novel and non-conserved known miRNAs are the main contributors to the difference (Wei et al., 2011). In pollen, small RNA displays cell-specific activity: working by translational repression in the SC, and by cleavage-induced mRNA turnover in the VC (Grant-Downton et al., 2013). The small RNA from the VC are strongly implicated in gene silencing in SCs (Slotkin et al., 2009; GrantDownton et al., 2013). This indicates reprogramming of gene expression during pollen development and the importance of epigenetic signals in this reprogramming. In addition, proteomics and metabolomics studies have revealed the importance of presynthesized proteins during pollen maturation in pollen function (Holmes-Davis et al., 2005; Dai et al., 2006), and difference in proteomes and metabolitic pathways between mature and germinated pollen (Dai et al., 2007; Obermeyer et al., 2013). These studies also revealed many important candidate genes for further understanding the molecular control of pollen development by functionally dissecting these candidates.

Recent studies have isolated SCs from tricellular pollen of rice and Arabidopsis and analyzed the transcriptome of SCs (Borges et al., 2008; Russell et al., 2012). The transcriptome of the SC was significantly different from that of the pollen grain, which is consistent with the SC being only a little part of the pollen grain that is mainly represented by the VC. SCpreferential transcripts showed a prominent functional skew toward epigenetic regulation, DNA repair, and cell cycles (Borges et al., 2008; Russell et al., 2012). Small RNA-mediated DNA methylation in SCs is associated with epigenetic inheritance, transposon silencing and paternal imprinting (Borges et al., 2008; Calarco et al., 2012). Further systematic "omics" analysis of molecular programs for SC development from its precursors, the GC and microspore, is essential to understand the mechanism of SC development. To achieve this goal, we need to establish a condition to isolate GCs and SCs from the pollen of a species. Because the GC occurs at a short time window in vivo and develops asynchronously in different flowers in rice and
Arabidopsis, isolating a large amount of GCs at high purity from developing pollen of these species for "omics" analysis is difficult.

Tomato is another model plant to study pollen development (Twell et al., 1990, 1991; Muschietti et al., 1994; Filichkin et al., 2004) and can be an excellent model to achieve the above target because (1) its genome has been sequenced (Sato et al., 2012) and (2) its mature pollen is bicellular. This feature of pollen indicates the possibility to isolate GCs from pollen grains or justgerminated pollen grains (JGPGs) and to isolate SCs from pollen tubes with SCs formed from GCs via mitosis.

In this study, we optimized the conditions of pollen germination and pollen tube growth to allow for long-term growth of pollen tubes in vitro with SCs generated in the tube. Using this culture system, we developed efficient protocols to isolate a large amount of GCs, SCs, and vegetative cell nuclei (VN) at high purity to satisfy the demands of "omics" study.

\section{Materials and Methods}

\section{Plants Growth and Pollen Collection}

Tomato (S. lycopersicum) plants (Heinz 1706) were grown in the greenhouse under long-day conditions (14 h light/10 h dark) at $25 \sim 35^{\circ} \mathrm{C}$. During anthesis, anthers from opened flowers were collected and dried for $10 \mathrm{~h}$ at $28^{\circ} \mathrm{C}$ in an electrothermal drying closet, then placed into a colander $(85 \mathrm{~mm} \times 50 \mathrm{~mm})$ with mesh at $63-\mu \mathrm{m}$-pore size; mature pollen was released and collected by shaking the colander vigorously. Pollen grains were used immediately or stored in a $1.5 \mathrm{~mL}$ tube with $5 \sim 10$ particles of Silica gel Rubin (Sigma, 85815) at $-20^{\circ} \mathrm{C}$.

\section{Pollen Germination In Vitro and Morphologic Observation}

Mature pollen grains $(60 \mathrm{mg})$ were pre-hydrated in a Petri dish $(60 \mathrm{~mm} \times 15 \mathrm{~mm})$, which was covered with gauze and then placed in a large Petri dish $(150 \mathrm{~mm} \times 25 \mathrm{~mm})$ with $50 \mathrm{~mL}$ saturated $\mathrm{Na}_{2} \mathrm{HPO}_{4}$ at $25^{\circ} \mathrm{C}$ for $4 \sim 8 \mathrm{~h}$. This device only permitted gauze contact this solution, and prohibited pollen grains contact the gauze and solution directly. Hydrated pollen grains were incubated in $100 \mathrm{~mL}$ germination medium $(20 \mathrm{mM}$ MES, $3 \mathrm{mM} \mathrm{Ca}\left(\mathrm{NO}_{3}\right)_{2}, 1 \mathrm{mM} \mathrm{KCl}, 0.8 \mathrm{mM} \mathrm{MgSO}_{4}, 1.6 \mathrm{mM}$ boric acid, 24\% PEG 4000, 2.5\% sucrose, $\mathrm{pH}$ 6.0; osmotic pressure, $\left.1253.33 \pm 2.33 \mathrm{mOsmol} / \mathrm{kg} \mathrm{H}_{2} \mathrm{O}\right)$ in a Petri dish $(150 \times 25 \mathrm{~mm})$ at $25^{\circ} \mathrm{C}$ in the dark with shaking at $90 \mathrm{rpm}$ (Tang et al., 2002; Zhao et al., 2013). During germination, $1 \mathrm{~mL}$ medium was took out at regular intervals, centrifuged to collect germinating pollen grains, then transferred to $1 \mathrm{~mL}$ Carnoy's fluid (three parts of absolute ethyl alcohol, one part of acetic acid) for treatment of $30 \mathrm{~min}$. Thereafter, these treated pollen grains or tubes were stained with 4',6-diamino-2-phenylindole (DAPI; Molecular Probes) and observed under a microscope (Zeiss Axio Imager A1).

\section{Isolation of GCs}

An improved two-step osmotic shock was used to release GCs from JGPGs and all procedures were performed at room 
temperature (Zhao et al., 2013). Two aliquots of pollen grains $90 \mathrm{mg}$ each were germinated in $100 \mathrm{~mL}$ germination medium as described above for $\sim 20$ min until the pollen tubes emerged but were shorter than the diameter of the grains. JGPGs were harvested through a Büchner funnel (100 $\mathrm{mm}$ in diameter) with 11- $\mu \mathrm{m}$ hydrated nylon mesh (Millipore, NY1100010) with the help of an aspirator pump, then rinsed with osmotic shock solution (15.3\% sucrose, $1 \%$ bovine serum albumin [BSA], $531.33 \pm 3.84 \mathrm{mOsmol} / \mathrm{kg} \mathrm{H}_{2} \mathrm{O}$ ) to clean germination medium, which would affect the result of osmotic shock. The collected JGPGs were immediately transferred to $80 \mathrm{~mL}$ fresh osmotic shock solution and incubated for $10 \mathrm{~min}$ to burst tubes and release GCs. Cell debris was removed by sieving the mixture through a hydrated $11-\mu \mathrm{m}$ nylon mesh. The filtrate containing GCs was equally divided into two centrifugation tubes, and centrifuged at $850 \mathrm{~g}$ for $4 \mathrm{~min}$ to collect GCs. To avoid loss of GCs, we retained $10 \mathrm{ml}$ of the supernatant in each tube after centrifugation, then added $10 \mathrm{~mL}$ of isolation buffer 1 (IB1; $20 \mathrm{mM}$ MES-KOH, $20 \mathrm{mM} \mathrm{NaCl}, 10 \mathrm{mM}$ EDTANa $_{2}, 1 \mathrm{mM}$ spermidine, $0.3 \mathrm{mM}$ spermine, $2 \mathrm{mM}$ DTT, $18 \%$ sucrose and $1 \%$ BSA, pH6.0) to suspend GCs. The suspension was supplemented with stock solution of cellulase "Onozuka" R-10 (Yakult) and macerozyme R-10 (Yakult; 0.4\% each in IB2; IB2; 10 mM MES$\mathrm{KOH}, 10 \mathrm{mM} \mathrm{NaCl}, 5 \mathrm{mM}$ EDTANa, $0.5 \mathrm{mM}$ spermidine, $0.15 \mathrm{mM}$ spermine, $1 \mathrm{mM}$ DTT, $18 \%$ sucrose and $1 \%$ BSA, pH6.0) to a final concentration of $0.04 \%$ each enzyme, mixed gently and incubated for 15 min without shaking, and centrifuged at $850 \mathrm{~g}$ for $3 \mathrm{~min}$ to collect GCs, followed by a washing with IB2. Collected GCs were further purified on 23/32\% Percoll density gradient $(2 \mathrm{~mL} \mathrm{23 \%} \mathrm{Percoll} \mathrm{and} 3 \mathrm{~mL} \mathrm{32 \%} \mathrm{Percoll} \mathrm{in}$ IB2) by horizontal centrifugation at $1000 \mathrm{~g}$ for $40 \mathrm{~min}$. After centrifugation, GCs partitioned at the interface of $23 \%$ and $32 \%$ Percoll were collected with use of a glass pipette and washed twice with $3 \times$ volume IB2 followed by centrifugation at $950 \mathrm{~g}$ for $3 \mathrm{~min}$ each. The viability of isolated GCs was examined by fluorescein diacetate (FDA) staining. The purified GCs were snap-frozen in liquid nitrogen and stored at $-80^{\circ} \mathrm{C}$.

\section{Isolation of SCs}

Sperm cells were isolated under room temperature as described by Xu et al. (2002) with modifications. In brief, three aliquots of pollen grains $60 \mathrm{mg}$ each were cultured in $100 \mathrm{~mL}$ germination medium as described above for $10 \mathrm{~h}$. After germination, medium was removed by use of hydrated $100-\mu \mathrm{m}$ nylon mesh (Millipore, NY1H00010), and pollen tubes were washed with osmotic shock solution as for isolation of GCs, immediately transferred to low-osmotic enzyme solution ( $0.4 \%$ cellulase "Onozuka" R-10 and $0.2 \%$ macerozyme R-10 in osmotic shock solution), and incubated for $5 \mathrm{~min}$ to release SCs. Cell debris and ungerminated pollen grains were removed by use of hydrated $11-\mu \mathrm{m}$ nylon mesh, and SCs in the filtrate were collected and washed as described in isolation of GCs. Thereafter, collected SCs were purified on $5 \mathrm{~mL} \mathrm{23 \%} \mathrm{Percoll} \mathrm{gradient} \mathrm{in} \mathrm{IB2} \mathrm{by} \mathrm{horizontal}$ centrifugation at $1000 \mathrm{~g}$ for $30 \mathrm{~min}$. SCs were enriched to the upper surface of $23 \%$ Percoll gradient and harvested by use of a glass pipette and washed twice with $3 \times$ volume IB2 followed by centrifugation at $950 \mathrm{~g}$ for $3 \mathrm{~min}$ each. The viability of isolated
SCs was examined by FDA staining. The purified SCs were snap-frozen in liquid nitrogen and stored at $-80^{\circ} \mathrm{C}$.

\section{Isolation of VN}

All operations were performed on ice or at $4^{\circ} \mathrm{C}$ unless otherwise specified, and all solutions were pre-cooled on ice or at $4^{\circ} \mathrm{C}$. Three aliquots of pollen grains $90 \mathrm{mg}$ each were cultured in $100 \mathrm{~mL}$ germination medium as described above for $1.5 \mathrm{~h}$. Pollen tubes were collected with a $20-\mu \mathrm{m}$ hydrated nylon mesh (Millipore, NY2009000) at room temperature, rinsed with wash buffer (10 mM MOPS-NaOH, 2.5\% sucrose, 9.5\% mannitol, $5 \mathrm{mM}$ EDTANa $_{2}, 1 \%$ BSA, pH7.2), and treated with $12 \mathrm{~mL}$ enzyme solution $(0.5 \%$ cellulase "Onozuka" $\mathrm{R}-10$, and $0.3 \%$ macerozyme $\mathrm{R}-10$ in wash buffer) for $5 \mathrm{~min}$ to release VN. After removal of ungerminated pollen grains and cell debris with use of $20-\mu \mathrm{m}$ hydrated nylon mesh, the filtrate containing $\mathrm{VN}$ was divided into four equal parts, loaded onto the surface of $3 \mathrm{~mL} 10 \%$ Percoll gradient in wash buffer each, then centrifuged at $1500 \mathrm{~g}$ for $30 \mathrm{~min}$. VN in the upper surface of the gradient were collected by use of a glass pipette, snap-frozen in liquid nitrogen, then stored at $-80^{\circ} \mathrm{C}$.

\section{Results}

\section{Dynamics of GCs and SCs During Culture In Vitro}

Our experiments showed that low-temperature $\left(-20^{\circ} \mathrm{C}\right)$ stored tomato pollen grains without prehydration germinated in vitro at low germination rate (Supplementary Table S1). To guarantee a high proportion of synchronously germinated tomato pollen grains after low-temperature storage and long-term growth of pollen tubes to allow generation of SCs in the tube in vitro, we optimized the pre-hydration condition of the stored pollen grains and culture condition of pollen tubes. We prehydrated low-temperature-stored tomato pollen grains using the saturated solution of $\mathrm{Na}_{2} \mathrm{HPO}_{4}$ to rescue the germination activity. Prehydration with the saturated solution for 4-8 h significantly increased germination rate of the pollen grains (Supplementary Table S1). Our culture conditions allowed for in vitro growth of pollen tubes for $>10 \mathrm{~h}$ (Figure 1).

To determine the suitable time of pollen tube growth for isolating GCs, SCs, and VN, we examined their dynamics during pollen germination and tube growth by DAPI staining. A bulge appeared at the germination aperture of hydrated pollen grains on culture for $20 \mathrm{~min}$, and the bulge emerged as a morphologically visible pollen tube with a length shorter than or equal to the diameter of the pollen grain during 30-min culture (Figure 1). With increased culture time, GCs and VN began to move into the tube at $1 \mathrm{~h}$ and completely entered the tube at $1.5 \mathrm{~h}$. We used DAPI staining to determine the movement order of $\mathrm{VN}$ and GCs (Figure 2). Among 126 surveyed pollen grains, for $68, \mathrm{VN}$ entered the tube first, and for 58, GCs entered first. Therefore, during tomato pollen tube growth, VN and GCs may move into the tube in a random order. Furthermore, for GCs, $77.8 \%$ completed mitosis to generate SCs at $8 \mathrm{~h}, 84.2 \%$ at $9 \mathrm{~h}$, and 92.4\% at $10 \mathrm{~h}$ (Supplementary Table S2). 


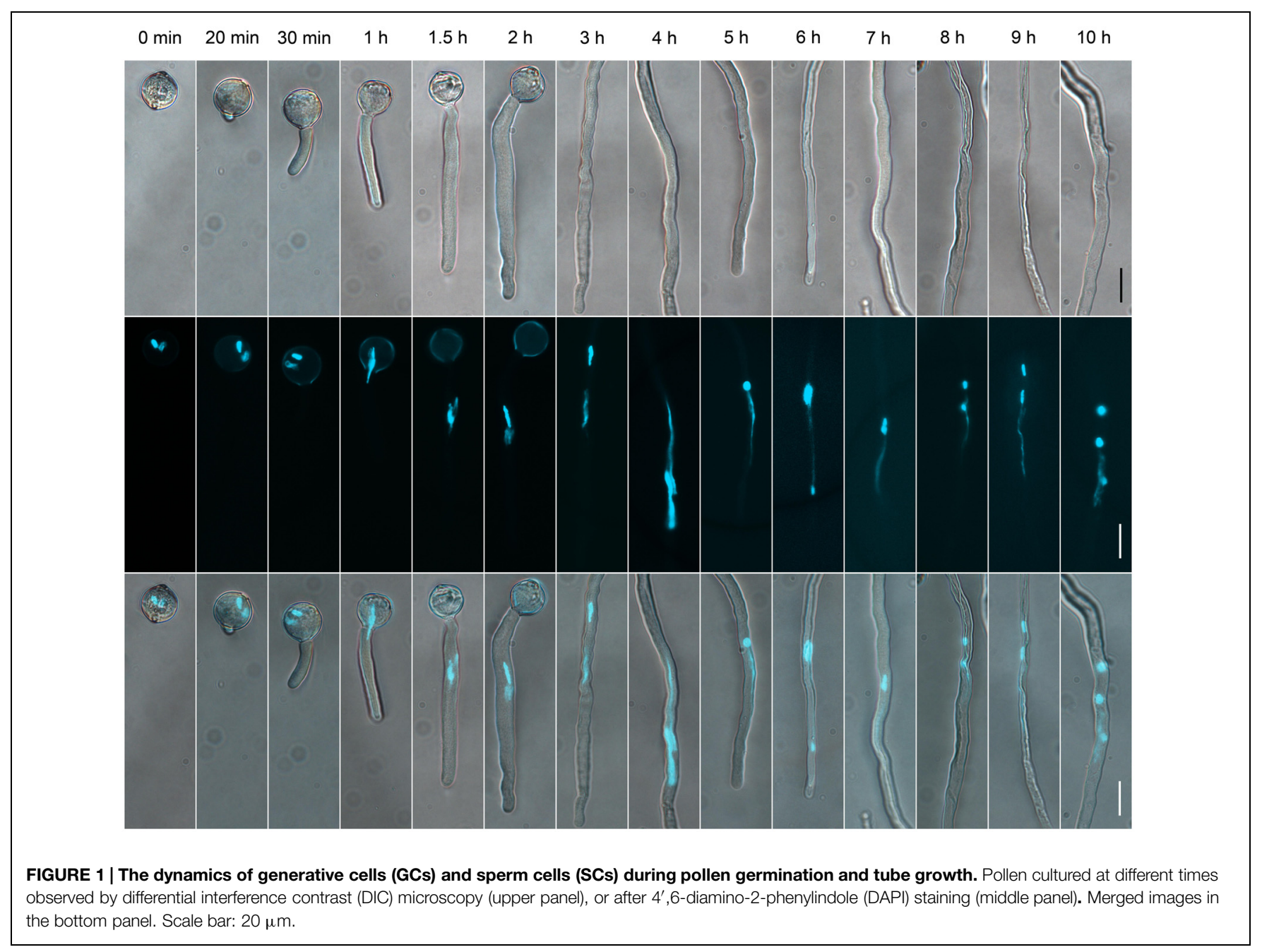

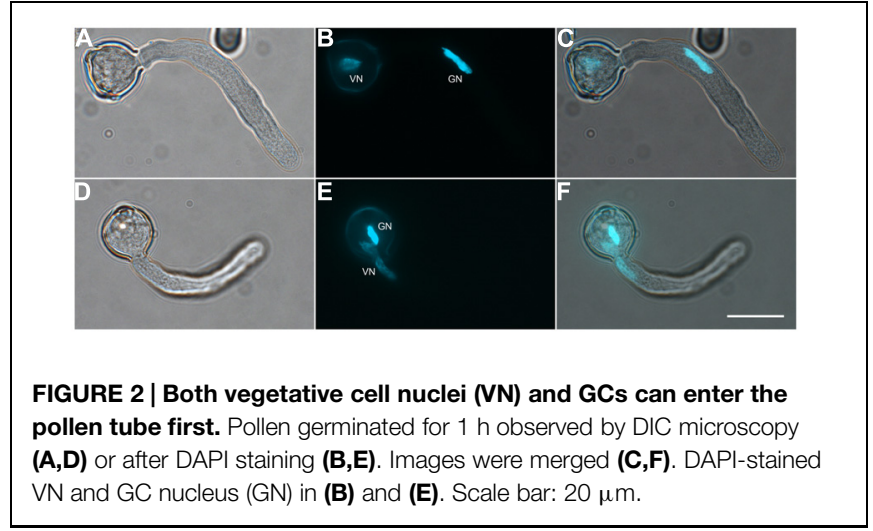

\section{Release and Purification of GCs}

Tomato pollen grains could not burst directly with osmotic shock and also could not germinate in a sucrose solution alone (data not shown). So, we developed a modified twostep method. We incubated prehydrated tomato pollen grains in germination medium for $20 \mathrm{~min}$, when a bulge appeared at the aperture (Figures 1 and $\mathbf{3 A}$ ), then osmotically shocked the JGPGs, which were sensitive to the low-osmotic shock (Figure 3B).

When JGPGs were transferred into low-osmotic solution, the tube burst, and GCs, along with the cytoplasm, were emitted (Figures 3C,D). The just-released GCs underwent a change from spindle- to oval-shaped (Figures 3C,D). This change may be associated with the microtubule cytoskeleton, which was dynamic in response to environmental conditions and is important to determine the shape of GCs (Zhou et al., 1990). When incubated in the low-osmotic solution for $10 \mathrm{~min}, 62.9 \%(680 / 1082)$ of pollen tubes burst (Figure 3B). The released GCs were intact in the low-osmotic solution up to $1 \mathrm{~h}$, but most GCs appeared to break after $1 \mathrm{~h}$ (data not shown). To maintain GC integrity, we added IB1 into the filtrate containing GCs to neutralize the low osmotic shock in a short time $(<1 \mathrm{~h})$. The suspension was used for purifying GCs.

Furthermore, we treated the suspension with cellulase and macerozyme at a low concentration, which had no effect on viability of GCs but increased the efficiency in removing cell debris at subsequent gradient centrifugation. GCs were enriched at the interface of $23 \%$ and $32 \%$ Percoll gradient, with cell debris on the upper interface of $23 \%$ Percoll. The isolated GCs 


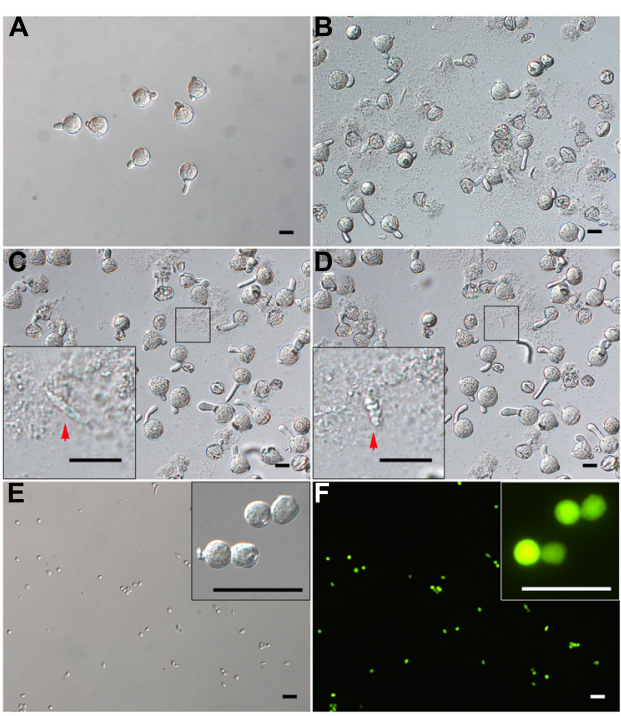

FIGURE 3 | Isolation of GCs from just-germinated pollen grains. (A) Pollen grains were geminated in vitro for $20 \mathrm{~min}$, and most produced pollen tubes. (B) 10-min osmotic shock of germinated pollen grains in osmotic shock solution led to a burst of most of pollen tubes. The inset image in (C) and (D) shows change of just-released GC from spindle-shaped (C) to oval-shaped (D). (E,F) DIC microscopy (E) and viability with fluorescein diacetate (FDA) staining (F) of purified GCs. Close-up of GCs in inset images (E,F). Scale bar: $20 \mu \mathrm{m}$.

were viable on FDA staining (Figures 3E,F) and had no VN contamination, as confirmed by propidium iodide (PI) staining (viable GCs cannot be stained by PI; Supplementary Figures S1A,B). Finally, we obtained about 1.5 million GCs from $180 \mathrm{mg}$ of initiated mature pollen grains (about 18 million grains, in that 0.1 million tomato pollen grains is about $1 \mathrm{mg}$ ).

\section{Release and Purification of SCs}

Successful isolation of SCs from in vitro-cultured pollen tubes depends on the formation of SCs in the growing tubes. We isolated SCs from 10-h-cultured pollen tubes, in which GCs had completed mitosis to generate SCs (see above, Figure 4A; Supplementary Table S2). To decrease the possible contamination of GCs, we collected long pollen tubes using a large pore-size nylon mesh $(100 \mu \mathrm{m})$, which allowed ungerminated pollen grains and shorter pollen tubes to pass through. We found that osmotic shock alone did not burst the long tubes efficiently (data not shown), and a modified low osmotic solution with cellulase and macerozyme was efficient to burst the tube (Figure 4B). After removal of cell debris, SCs in filtrate could be enriched with a layer of $23 \%$ Percoll. Finally, we obtained about 2 million viable SCs at high purity from $180 \mathrm{mg}$ initiated pollen grains (Figures 4C,D), with no VN contamination (Supplementary Figures S1C,D).

\section{Release and Purification of VN}

Our results showed that VN was fragile and disrupted quickly as released to medium at room temperature. Repeated pipetting also

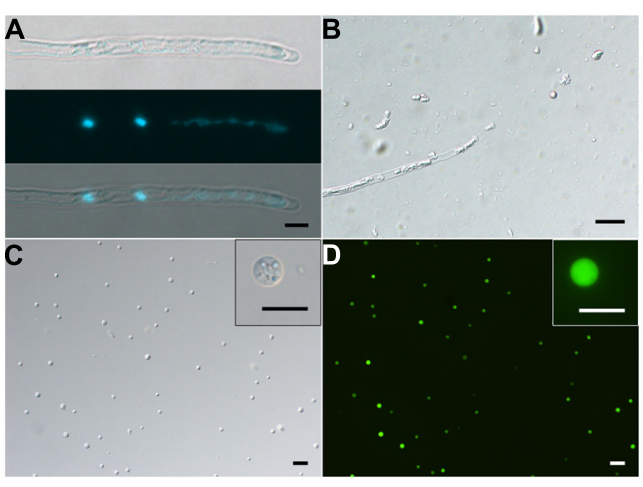

FIGURE 4 | Isolation of SCs from 10-h-cultured pollen tubes. (A) DIC (upper) and DAPI staining (middle) and merged (bottom) images of pollen tube. (B) Representative enzymolysis-treated pollen tubes. (C,D) DIC microscopy (C) and viability of purified SCs with FDA staining (D). The inset images in (C) and (D) are close-ups of the SC. Scale bar: $20 \mu \mathrm{m}$ in (A) to (D), $10 \mu \mathrm{m}$ in inset images of (C) and (D).

led to its complete disruption (Supplementary Figures S2A,B,C). Therefore, no $\mathrm{VN}$ contamination was present in isolated GCs and SCs (see above). We solved the bottleneck of $\mathrm{VN}$ isolation by (1) keeping all operations at $4^{\circ} \mathrm{C}$ or on ice, (2) avoiding pipetting as much as possible, and (3) using 1.5-h-cultured pollen tubes in which $\mathrm{VN}$ had moved into the tube (Figures 1 and 5A,B), for easier release of VN (Figures 5C,D). Furthermore, additional washing as well as passing through Percoll on gradient centrifugation disrupted VN (Supplementary Figures S2A,D), so we used only a hydrated nylon net filter to remove pollen grains and cell debris and then enriched VN by using a layer of $10 \%$ Percoll. These measures allowed for isolation of VN without GC contamination (Figures 5E,F). Using this protocol, we obtained 10 million $\mathrm{VN}$ from $270 \mathrm{mg}$ initiated pollen grains.

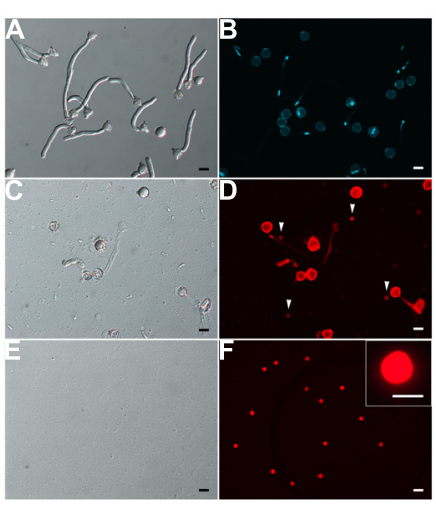

FIGURE 5 | Isolation of VN from pollen tubes. (A,B) DIC (A) and DAPI staining (B) of pollen tubes cultured in vitro for $1.5 \mathrm{~h}$. (C,D) DIC microscopy (C) and propodium iodide (PI) staining (D) of enzymolysis-treated pollen tubes; arrows indicate released VNs (D). (E,F) DIC microscopy (E) and PI staining (F) of purified VN. Inset image in $(\mathbf{F})$ is close-up of a Pl-stained VN. Scale bar: $20 \mu \mathrm{m}$ in $(\mathbf{A})$ to $(\mathbf{F}), 10 \mu \mathrm{m}$ in the inset image (F). 


\section{Discussion}

We have optimized the conditions allowing for growth of pollen tubes for more than $10 \mathrm{~h}$ and generation of SCs in tubes, as well as conditions affecting rupture of pollen grains (tubes) and release of cytoplasm, GCs, SCs and VN into medium. Finally, we developed methods to isolate GCs, SCs, and VN from JGPGs and 1.5-hand 10-h-cultured pollen tubes, respectively (Figure 6). These methods allowed for isolating large amounts of GCs, SCs, and VN at high purity.

\section{Culture Conditions for Low-Temperature-Stored Pollen Grains and Long-Term-Cultured Pollen Tubes}

Previous study established the condition for in vitro germination of fresh pollen grains from tomato (Tang et al., 2002), but under the condition, low-temperature-stored tomato pollen grains did not germinate well (Supplementary Table S1). Generally, pre-hydration is required for rescuing the viability of lowtemperature-stored pollen grains, such as from Rosa, Pistacia vera L., Gladiolus sp. and Brassica rapa (Visser et al., 1977; GolanGoldhirsh et al., 1991; Rajasekharan et al., 1994; Sato et al., 1998). The prehydration was usually actualized with water or saturated salt solution, which generated a fixed relative humidity in a chamber at a certain temperature. We found that a saturated solution of $\mathrm{Na}_{2} \mathrm{HPO}_{4}$ was suitable for prehydrating tomato pollen grains. The saturated solution was previously used to prehydrate B. rapa pollen (Sato et al., 1998), and could result in $95 \%$ relative humidity in a chamber at $25^{\circ} \mathrm{C}$, and tomato pollen grains rescued

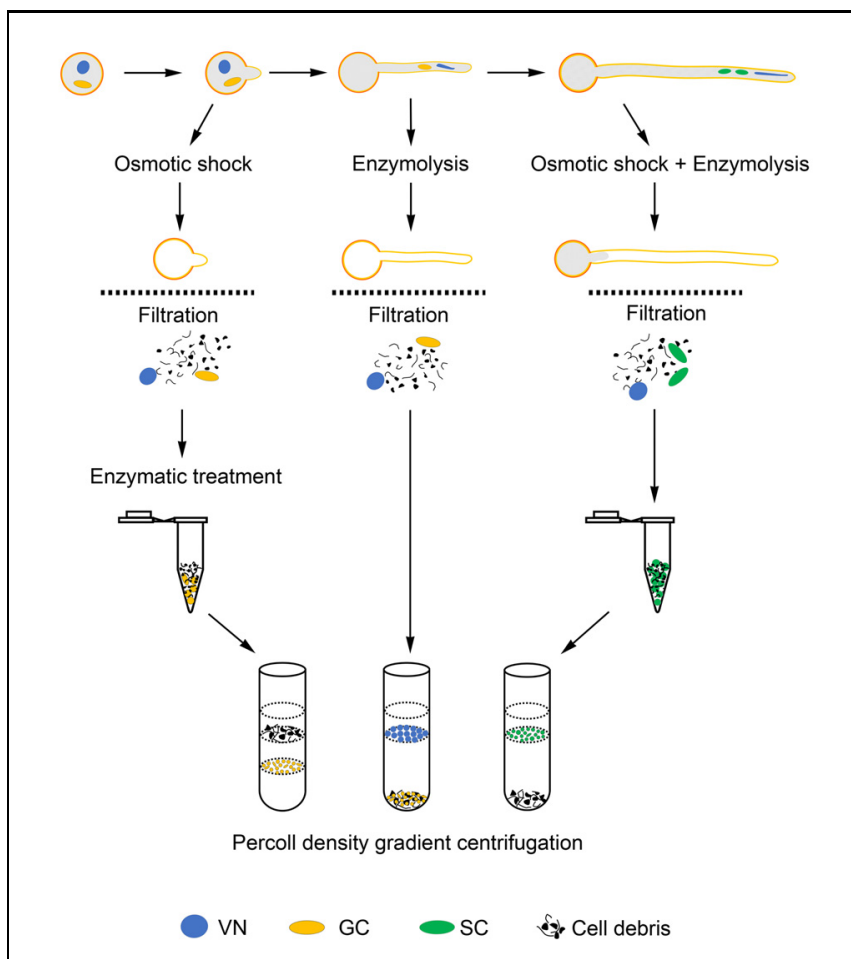

FIGURE 6 | Schematic of methods to isolate GCs, SCs, and VN. under this condition germinated synchronously (Figure 3A), which was important for synchronous pollen tube growth and GC division. Pollen density also was an important factor affecting germination in tomato, and increased density led to increased germination percentage (Supplementary Figure S3), which agrees with observations in other species such as Arabidopsis (Boavida and McCormick, 2007), Nicotiana, B. oleracea, and Betula pendula (Roberts et al., 1983; Jahnen et al., 1989; Pasonen and Käpylä, 1998; Chen et al., 2000). Furthermore, we evaluated the effect of loaded pollen grain amount in a given volume germination medium on the integrity of pollen tubes during longterm culture $(>3 \mathrm{~h})$ by examining cell debris in the medium (Supplementary Figure S4). Cell debris was barely observed with $\leq 4 \mathrm{mg}$ pollen grains used (Supplementary Figures S4A-D), and substantial with $5 \mathrm{mg}$ pollen grains (Supplementary Figure S4E). Thus, the amount of pollen grains in a given volume germination medium is crucial to the integrity of long-term-cultured pollen tubes, and $3 \mathrm{mg}$ pollen grains loaded in $5 \mathrm{~mL}$ germination medium was appropriate for long-term culture of pollen tubes.

\section{Methods to Release GCs, SCs, and VN}

Four major methods were used previously to break pollen grains or tubes: mechanical grinding, one-step and two-step osmotic shock and enzyme digestion (Russell, 1986, 1991; Zhou, 1988; Russell et al., 1990; Theunis et al., 1991; Chaboud and Perez, 1992; Xu et al., 2002; Borges et al., 2008; Zhao et al., 2013). The mechanical grinding had relatively low efficiency for breaking tomato pollen grains and produced a large amount of debris, which interfered with further purification. One-step or two-step osmotic shock is usually used to break pollen grains (tubes) and release target cells. The former breaks pollen grains and releases target cells simultaneously using a low-osmotic solution (Russell, 1986). The latter first makes the grain germinate in a sucrose solution, then release target cells under low-osmotic shock with a diluted sucrose solution (Zhou, 1988; Wu and Zhou, 1991). Tomato pollen grains were insensitive to osmotic shock and even did not burst under osmotic shock of water, which is similar to pollen grains from Vicia faba, B. napus, and $L$. davidii var. unicdor (Zhou, 1988; Taylor et al., 1991; Zhao et al., 2013), thus suggesting a complicated mechanism of pollen grain burst for different species and individual methods needed for different species.

We choose the two-step osmotic shock to release GCs. Components of osmotic shock solution affected the appearance of released cytoplasm, which affected the following purification of GCs. The released cytoplasm appeared not to conglutinate when the solution had only $15.3 \%$ sucrose (Supplementary Figure S5A) but appeared to conglutinate with shock solution containing MES or MOPS regardless of concentration (Supplementary Figures S5B-I). Acidic pH could aggravate this situation (Supplementary Figures S5D,J,L). The phenomenon of cytoplasm clumping was also observed under high concentration of $\mathrm{CaCl}_{2}$ in a previous study of isolating SCs from pollen tubes of $N$. tabacum (Tian and Russell, 1997). We chose sucrose and BSA as the components of the osmotic shock solution. The use of BSA in all solutions 
except the germination medium aimed to protect GCs against damage because we found that GCs could remain intact in the shock solution with BSA up to $1 \mathrm{~h}$ but for only a few minutes in a shock solution without BSA; GCs from several species such as $V$. faba could remain intact in simple sucrose shock solution until being purified (Zhou, 1988).

However, pollen tubes cultured in vitro for $10 \mathrm{~h}$, which we used to isolate SCs, were not as sensitive as pollen tubes cultured for a short time to osmotic shock described above. So we developed a modified shock solution containing cellulase and macroenzyme. The two enzymes are usually applied to digest hemicelluloses, cellulose and pectin, the main components of the pollen tube wall (Taylor and Hepler, 1997; Cheung and Wu, 2008; Rounds and Bezanilla, 2013). This modified shock solution broke the long pollen tubes and released SCs efficiently (Figure 4B). Why pollen tubes had different sensitivity to osmotic shock with long- and short-term culture needs further studies.

In contrast to reports of GCs and SCs, we have limited reports of VN isolation (Wever and Takats, 1971; LaFountain and Mascarenhas, 1972; Ueda and Tanaka, 1994; Borges et al., 2012; Calarco et al., 2012). These reports did not describe the stability of isolated $\mathrm{VN}$ or the effect of environmental conditions on the stability. We found that VN was fragile in vitro (Supplementary Figure S2) and easily ruptured at room temperature but was relatively stable at $4^{\circ} \mathrm{C}$. However, at low temperature, pollen tubes were not sensitive to osmotic shock. In this situation, enzyme digestion was found efficient to break pollen tubes and release VN but depended on a suitable buffer. We found that $\mathrm{pH}$ value was a crucial factor of the buffer. The $\mathrm{pH}$ value affected the appearance of the released cytoplasm: conglutination appeared at acidic $\mathrm{pH}$ and not at alkalinous $\mathrm{pH}$ (Supplementary Figure S6).

\section{Measures to Guarantee the Purity of GCs, SCs, and VN}

Previous reports mainly described isolation of SCs from tricellular pollen or GCs from bicellular pollen (Russell, 1986; Dupuis et al., 1987; Zhou, 1988; Southworth and Knox, 1989; Yang and Zhou, 1989; Xu et al., 2002; Engel et al., 2003), or along with VN (Borges et al., 2012). For these cases, possible contaminants were VN for isolated SCs or GCs and vice versa. Such purity evaluation was relatively simple. Most previous works evaluated the purity of isolated SCs or GCs based on their morphologic features observed on microscopy (Zhou, 1988; Southworth and Knox, 1989; Yang and Zhou, 1989; Xu et al., 2002; Engel et al., 2003). Only the study of Arabidopsis used SCand VN-specific markers to estimate the purity of isolated SCs and $\mathrm{VN}$ because of available markers for this species (Borges et al., 2008, 2012).

An evaluation of the purity of isolated GCs, SCs, or VN from a species was relatively lacking in early studies. We found that a combination of PI staining and differential interference contrast (DIC) microscope observation was efficient to evaluate VN contamination of GCs or SCs and vice versa. VN was not observed on DIC microscopy but were detectable with PI staining. In contrast, viable GCs and SCs were easily observed on DIC microscopy but undetectable with PI staining. Using the combination, we did not find $\mathrm{VN}$ contamination in isolated GCs and SCs or GC and SC contamination in isolated VN (Supplementary Figure S1, Figures 5E,F). We considered several measures to eliminate this contamination in designing methods to isolate GCs, SCs, and VN. (1) GCs or SCs were stable and isolated at room temperature, but under this temperature, VN was fragile and broke quickly. (2) GCs, SCs and VN had different density on Percoll gradient and could be enriched with different gradient ingredients. These measures could eliminate VN contamination in isolated GCs and SCs and vice versus (Supplementary Figure S1, Figures 5E,F).

A major challenge in methodology was how to get SCs at high purity. Here, besides the use of high synchronous pollen tubes, of which $92.4 \%$ generated SCs (Supplementary Table S2), and the measures above, we collected long pollen tubes by using a large-pore mesh (100 $\mu \mathrm{m}$ in pore diameter), which excluded ungerminated pollen grains (diameter of about $20 \mu \mathrm{m}$ ) and short pollen tubes. Thus, the methods could obtain SCs at high purity. However, tools to distinguish GCs and SCs are lacking because of their similar morphology under light microscope in tomato and other most species and lack of molecular markers.

\section{Conclusion}

Saturated $\mathrm{Na}_{2} \mathrm{HPO}_{4}$ solution was suitable for pre-hydration of low-temperature-stored tomato pollen grains, and the prehydrated pollen grains germinated synchronously. The loaded amount of $0.6 \mathrm{mg}$ pollen grains per $\mathrm{mL}$ allowed pollen tubes to grow for more than $10 \mathrm{~h}$, and more than $92 \%$ GCs completed mitosis to generate SCs. GCs or SCs were stable and could be isolated at room temperature, whereas under the same temperature, VN was fragile and broke quickly in vitro. GCs, SCs, and VN had different density on Percoll gradient, and could be enriched with different gradient ingredients. Thus, we have established methods to isolate GCs and VN from just-germinated pollen grains and 1.5-h-cultured pollen tubes, respectively, and SCs from 10-h-cultured pollen tubes. Using these methods, we could obtain 1.5 million GCs and 2 million SCs each from $180 \mathrm{mg}$ initiated pollen grains, and 10 million VN from $270 \mathrm{mg}$ initiated pollen grains, for higher productivity as compared with previous reports of other species.

\section{Acknowledgments}

We thank Xin Zhao for help in developing these methods, and Fan Yang, Bo Yu, Yunyun Song, Yuxia Liu for help in collecting tomato pollen grains. This work was supported by the Chinese Ministry of Science and Technology (grant no. 2012CB910504).

\section{Supplementary Material}

The Supplementary Material for this article can be found online at: http://journal.frontiersin.org/article/10.3389/fpls. 2015.00391/abstract 


\section{References}

Berger, F., and Twell, D. (2011). Germline specification and function in plants. Annu. Rev. Plant Biol. 62, 461-484. doi: 10.1146/annurev-arplant-042110103824

Boavida, L. C., and McCormick, S. (2007). Temperature as a determinant factor for increased and reproducible in vitro pollen germination in Arabidopsis thaliana. Plant J. 52, 570-582. doi: 10.1111/j.1365-313X.2007.03248.x

Borg, M., Brownfield, L., Khatab, H., Sidorova, A., Lingaya, M., and Twell, D. (2011). The R2R3 MYB transcription factor DUO1 activates a male germlinespecific regulon essential for sperm cell differentiation in Arabidopsis. Plant Cell 23, 534-549. doi: 10.1105/tpc.110.081059

Borg, M., Brownfield, L., and Twell, D. (2009). Male gametophyte development: a molecular perspective. J. Exp. Bot. 60, 1465-1478. doi: 10.1093/Jxb/Ern355

Borg, M., Rutley, N., Kagale, S., Hamamura, Y., Gherghinoiu, M., Kumar, S., et al. (2014). An EAR-dependent regulatory module promotes male germ cell division and sperm fertility in Arabidopsis. Plant Cell 26, 2098-2113. doi: 10.1105/tpc.114.124743

Borges, F., Gardner, R., Lopes, T., Calarco, J. P., Boavida, L. C., Slotkin, R. K., et al. (2012). FACS-based purification of Arabidopsis microspores, sperm cells and vegetative nuclei. Plant Methods 8:44. doi: 10.1186/17464811-8-44

Borges, F., Gomes, G., Gardner, R., Moreno, N., McCormick, S., Feijó, J. A., et al. (2008). Comparative transcriptomics of Arabidopsis sperm cells. Plant Physiol. 148, 1168-1181. doi: 10.1104/pp.108.125229

Brownfield, L., Hafidh, S., Borg, M., Sidorova, A., Mori, T., and Twell, D. (2009a). A plant germline-specific integrator of sperm specification and cell cycle progression. PLoS Genet. 5:e1000430. doi: 10.1371/journal.pgen. 1000430

Brownfield, L., Hafidh, S., Durbarry, A., Khatab, H., Sidorova, A., Doerner, P., et al. (2009b). Arabidopsis DUO POLLEN3 is a key regulator of male germline development and embryogenesis. Plant Cell 21, 1940-1956. doi: $10.1105 /$ tpc. 109.066373

Calarco, J. P., Borges, F., Donoghue, M. T., Van Ex, F., Jullien, P. E., Lopes, T., et al. (2012). Reprogramming of DNA methylation in pollen guides epigenetic inheritance via small RNA. Cell 151, 194-205. doi: 10.1016/j.cell.2012. 09.001

Chaboud, A., and Perez, R. (1992). Generative cells and male gametes: isolation, physiology, and biochemistry. Int. Rev. Cytol. 140, 205-232. doi: 10.1016/S00747696(08)61098-0

Chen, Y. F., Matsubayashi, Y., and Sakagami, Y. (2000). Peptide growth factor phytosulfokine- $\alpha$ contributes to the pollen population effect. Planta 211, 752 755. doi: $10.1007 / \mathrm{s} 004250000370$

Cheung, A. Y., and Wu, H. M. (2008). Structural and signaling networks for the polar cell growth machinery in pollen tubes. Annu. Rev. Plant Biol. 59, 547-572. doi: 10.1146/annurev.arplant.59.032607.092921

Dai, S. J., Chen, T. T., Chong, K., Xue, Y. B., Liu, S. Q., and Wang, T. (2007). Proteomics identification of differentially expressed proteins associated with pollen germination and tube growth reveals characteristics of germinated Oryza sativa pollen. Mol. Cell. Proteomics 6, 207-230. doi: 10.1074/mcp.M600146MCP200

Dai, S., Li, L., Chen, T., Chong, K., Xue, Y., and Wang, T. (2006). Proteomic analyses of Oryza sativa mature pollen reveal novel proteins associated with pollen germination and tube growth. Proteomics 6, 2504-2529. doi: 10.1002/pmic.200401351

Dupuis, I., Roeckel, P., Matthys-Rochon, E., and Dumas, C. (1987). Procedure to isolate viable sperm cells from corn (Zea mays L.) pollen grains. Plant Physiol. 85, 876-878. doi: 10.1104/pp.85.4.876

Engel, M. L., Chaboud, A., Dumas, C., and McCormick, S. (2003). Sperm cells of Zea mays have a complex complement of mRNAs. Plant J. 34, 697-707. doi: 10.1046/j.1365-313X.2003.01761.x

Filichkin, S. A., Leonard, J. M., Monteros, A., Liu, P. P., and Nonogaki, H. (2004). A novel endo- $\beta$-mannanase gene in tomato LeMAN5 is associated with anther and pollen development. Plant Physiol. 134, 1080-1087. doi: 10.1104/pp.103.035998

Golan-Goldhirsh, A., Schmidhalter, U., Müller, M., and Oertli, J. J. (1991). Germination of Pistacia vera L. pollen in liquid medium. Sex. Plant Reprod. 4, 182-187. doi: 10.1007/BF00190002
Grant-Downton, R., Kourmpetli, S., Hafidh, S., Khatab, H., Le Trionnaire, G. Dickinson, H., et al. (2013). Artificial microRNAs reveal cell-specific differences in small RNA activity in pollen. Curr. Biol. 23, R599-R601. doi: 10.1016/j.cub.2013.05.055

Holmes-Davis, R., Tanaka, C. K., Vensel, W. H., Hurkman, W. J., and McCormick, S. (2005). Proteome mapping of mature pollen of Arabidopsis thaliana. Proteomics 5, 4864-4884. doi: 10.1002/pmic.200402011

Honys, D., and Twell, D. (2004). Transcriptome analysis of haploid male gametophyte development in Arabidopsis. Genome Biol. 5, R85. doi: 10.1186/Gb-2004-5-11-R85

Jahnen, W., Lush, W. M., and Clarke, A. E. (1989). Inhibition of in vitro pollen tube growth by isolated S-glycoproteins of Nicotiana alata. Plant Cell 1, 501-510. doi: $10.1105 /$ tpc.1.5.501

LaFountain, K. L., and Mascarenhas, J. P. (1972). Isolation of vegetative and generative nuclei from pollen tubes. Exp. Cell Res. 73, 233-236. doi: 10.1016/0014-4827(72)90125-5

McCormick, S. (1993). Male gametophyte development. Plant Cell 5, 1265-1275. doi: $10.2307 / 3869779$

Muschietti, J., Dircks, L., Vancanneyt, G., and McCormick, S. (1994). Lat52 protein is essential for tomato pollen development: pollen expressing antisense Lat52 RNA hydrates and germinates abnormally and cannot achieve fertilization. Plant J. 6, 321-338. doi: 10.1046/j.1365-313X.1994.06030321.x

Obermeyer, G., Fragner, L., Lang, V., and Weckwerth, W. (2013). Dynamic adaption of metabolic pathways during germination and growth of lily pollen tubes after inhibition of the electron transport chain. Plant Physiol. 162, 18221833. doi: $10.1104 /$ pp. 113.219857

Pasonen, H. L., and Käpylä, M. (1998). Pollen-pollen interactions in Betula pendula in vitro. New Phytol. 138, 481-487. doi: 10.1046/j.1469-8137.1998.00135.x

Rajasekharan, P. E., Rao, T. M., Janakiram, T., and Ganeshan, S. (1994). Freeze preservation of gladiolus pollen. Euphytica 80, 105-109. doi: 10.1007/Bf00039304

Roberts, I. N., Gaude, T. C., Harrod, G., and Dickinson, H. G. (1983). Pollen-stigma interactions in Brassica oleracea; a new pollen germination medium and its use in elucidating the mechanism of self incompatibility. Theor. Appl. Genet. 65 231-238. doi: 10.1007/Bf00308074

Rounds, C. M., and Bezanilla, M. (2013). Growth mechanisms in tip-growing plant cells. Annu. Rev. Plant Biol. 64, 243-265. doi: 10.1146/annurev-arplant-050312120150

Russell, S. D. (1986). Isolation of sperm cells from the pollen of Plumbago zeylanica. Plant Physiol. 81, 317-319. doi: 10.1104/pp.81.1.317

Russell, S. D. (1991). Isolation and characterization of sperm cells in flowering plants. Annu. Rev. Plant Physiol. Plant Mol. Biol. 42, 189-204. doi: 10.1146/annurev.arplant.42.1.189

Russell, S. D., Cresti, M., and Dumas, C. (1990). Recent progress on sperm characterization in flowering plants. Physiol. Plant. 80, 669-676. doi: 10.1034/j.1399-3054.1990.800427.x

Russell, S. D., Gou, X. P., Wong, C. E., Wang, X. K., Yuan, T., Wei, X. P., et al. (2012). Genomic profiling of rice sperm cell transcripts reveals conserved and distinct elements in the flowering plant male germ lineage. New Phytol. 195, 560-573. doi: 10.1111/j.1469-8137.2012.04199.x

Rutley, N., and Twell, D. (2015). A decade of pollen transcriptomics. Plant Reprod. 28, 73-89. doi: 10.1007/s00497-015-0261-7

Sato, S., Katoh, N., Iwai, S., and Hagimori, M. (1998). Establishment of reliable methods of in vitro pollen germination and pollen preservation of Brassica rapa (syn. B campestris). Euphytica 103, 29-33. doi: 10.1023/A:1018381417657.

Sato, S., Tabata, S., Hirakawa, H., Asamizu, E., Shirasawa, K., Isobe, S., et al. (2012). The tomato genome sequence provides insights into fleshy fruit evolution. Nature 485, 635-641. doi: 10.1038/Nature11119

Slotkin, R. K., Vaughn, M., Borges, F., Tanurdžić, M., Becker, J. D., Feijó, J. A., et al. (2009). Epigenetic reprogramming and small RNA silencing of transposable elements in pollen. Cell 136, 461-472. doi: 10.1016/j.cell.2008.12.038

Southworth, D., and Knox, R. B. (1989). Flowering plant sperm cells: isolation from pollen of Gerbera jamesonii (Asteraceae). Plant Sci. 60, 273-277. doi: 10.1016/0168-9452(89)90177-5

Tang, W. H., Ezcurra, I., Muschietti, J., and McCormick, S. (2002). A cysteinerich extracellular protein, LAT52, interacts with the extracellular domain of the pollen receptor kinase LePRK2. Plant Cell 14, 2277-2287. doi: $10.1105 /$ Tpc.003103 
Taylor, L. P., and Hepler, P. K. (1997). Pollen germination and tube growth. Annu. Rev. Plant Physiol. Plant Mol. Biol. 48, 461-491. doi: 10.1146/annurev.arplant.48.1.461

Taylor, P. E., Kenrick, J., Blomstedt, C. K., and Knox, R. B. (1991). Sperm cells of the pollen tubes of Brassica: ultrastructure and isolation. Sex. Plant Reprod. 4, 226-234. doi: 10.1007/BF00190009

Theunis, C. H., Pierson, E. S., and Cresti, M. (1991). Isolation of male and female gametes in higher plants. Sex. Plant Reprod. 4, 145-154. doi: 10.1007/BF00189998

Tian, H. Q., and Russell, S. D. (1997). Micromanipulation of male and female gametes of Nicotiana tabacum 1. Isolation of gametes. Plant Cell Rep. 16, 555-560. doi: 10.1007/BF01142323

Twell, D. (2011). Male gametogenesis and germline specification in flowering plants. Sex. Plant Reprod. 24, 149-160. doi: 10.1007/s00497-010-0157-5

Twell, D., Park, S. K., and Lalanne, E. (1998). Asymmetric division and cellfate determination in developing pollen. Trends Plant Sci. 3, 305-310. doi: 10.1016/S1360-1385(98)01277-1

Twell, D., Yamaguchi, J., and McCormick, S. (1990). Pollen-specific gene expression in transgenic plants: coordinate regulation of two different tomato gene promoters during microsporogenesis. Development 109 , 705-713.

Twell, D., Yamaguchi, J., Wing, R. A., Ushiba, J., and McCormick, S. (1991). Promoter analysis of genes that are coordinately expressed during pollen development reveals pollen-specific enhancer sequences and shared regulatory elements. Genes Dev. 5, 496-507. doi: 10.1101/Gad.5.3.496

Ueda, K., and Tanaka, I. (1994). The basic proteins of male gametic nuclei isolated from pollen grains of Lilium longiflorum. Planta 192, 446-452. doi: 10.1007/BF00198582

Visser, T., De Vries, D. P., Welles, G. W. H., and Scheurink, J. A. M. (1977). Hybrid tea-rose pollen. I. Germination and storage. Euphytica 26, 721-728. doi: 10.1007/Bf00021697

Wang, Y., Zhang, W. Z., Song, L. F., Zou, J. J., Su, Z., and Wu, W. H. (2008). Transcriptome analyses show changes in gene expression to accompany pollen germination and tube growth in Arabidopsis. Plant Physiol. 148, 1201-1211. doi: 10.1104/pp.108.126375

Wei, L. Q., Xu, W. Y., Deng, Z. Y., Su, Z., Xue, Y. B., and Wang, T. (2010). Genomescale analysis and comparison of gene expression profiles in developing and germinated pollen in Oryza sativa. BMC Genomics 11:338. doi: 10.1186/1471 2164-11-338

Wei, L. Q., Yan, L. F., and Wang, T. (2011). Deep sequencing on genome-wide scale reveals the unique composition and expression patterns of microRNAs in developing pollen of Oryza sativa. Genome Biol. 12:R53. doi: 10.1186/Gb-201112-6-R53

Wever, G. H., and Takats, S. T. (1971). Isolation and separation of S-competent and S-incompetent nuclei from Tradescantia pollen grains. Exp. Cell Res. 69, 29-32. doi: 10.1016/0014-4827(71)90306-5

Wu, X., and Zhou, C. (1991). A comparative study on methods for isolation of generative cell in various angiosperm species. Acta Biol. Exp. Sin. 24, 15-23.

$\mathrm{Xu}$, H. P., Weterings, K., Vriezen, W., Feron, R., Xue, Y. B., Derksen, J., et al. (2002). Isolation and characterization of male-germ-cell transcripts in Nicotiana tabacum. Sex. Plant Reprod. 14, 339-346. doi: 10.1007/s00497-0020128-6

Yang, H. Y., and Zhou, C. (1989). Isolation of viable sperms from pollen of Brassics napus, Zea mays and Secale cereale. Chin. J. Bot. 1, 80-84.

Zhao, X., Yang, N., and Wang, T. (2013). Comparative proteomic analysis of generative and sperm cells reveals molecular characteristics associated with sperm development and function specialization. J. Proteome Res. 12, 5058-5071. doi: 10.1021/pr400291p

Zhou, C. (1988). Isolation and purification of generative cells from fresh pollen of Vicia faba L. Plant Cell Rep. 7, 107-110. doi: 10.1007/BF00270116

Zhou, C., Zee, S.Y., and Yang, H. Y. (1990). Microtubule organization of in situ and isolated generative cells in Zephyranthes granditlora Lindl. Sex. Plant Reprod. 3, 213-218. doi: 10.1007/BF00202877

Conflict of Interest Statement: The authors declare that the research was conducted in the absence of any commercial or financial relationships that could be construed as a potential conflict of interest.

Copyright (c) $2015 \mathrm{Lu}$, Wei and Wang. This is an open-access article distributed under the terms of the Creative Commons Attribution License (CC BY). The use, distribution or reproduction in other forums is permitted, provided the original author(s) or licensor are credited and that the original publication in this journal is cited, in accordance with accepted academic practice. No use, distribution or reproduction is permitted which does not comply with these terms. 